\title{
Analysis of continuously-rotating quadrupole focusing channels using generalized Courant-Snyder theory
}

\author{
Moses Chung \\ Accelerator Physics Center, Fermi National Accelerator Laboratory, Batavia, IL 60510 \\ Hong Qin, Erik Gilson, and Ronald C. Davidson \\ Plasma Physics Laboratory, Princeton University, Princeton, NJ 08543
}

(Dated: July 5, 2013)

\begin{abstract}
By extending the recently developed generalized Courant-Snyder theory for coupled transverse beam dynamics, we have constructed the Gaussian beam distribution and its projections with arbitrary mode emittance ratios. The new formulation has been applied to a continuously-rotating quadrupole focusing channel because the basic properties of this channel are known theoretically and could also be investigated experimentally in a compact setup such as the linear Paul trap configuration. The new formulation retains a remarkably similar mathematical structure to the original Courant-Snyder theory, and thus provides a powerful theoretical tool to investigate coupled transverse beam dynamics in general and more complex linear focusing channels.
\end{abstract}




\section{INTRODUCTION}

The well-known Courant-Snyder (CS) theory [1] provides a fundamental framework for the uncoupled transverse dynamics of charged particles in linear focusing lattices. The CS theory has been extensively used to design and analyze alternating-gradient focusing systems for more than 50 years. Various attempts to generalize the CS theory to the case of coupled transverse dynamics with two or more degrees of freedom have been made in the past [26]. The method recently developed by Qin and Davidson [7,8] is particulary noteworthy because, unlike other methods, it retains mathematical structures remarkably similar to the original CS theory. The envelope function is generalized into an envelope matrix, and the phase advance is generalized into a 4D symplectic rotation.

The generalized CS theory was successfully applied to describe beam envelopes of equal emittances in two normal planes for the cases of Gaussian [9] and Kapchinskij-Vladimirskij $(\mathrm{KV})[10]$ distributions. For the equal emittance case, the beam matrix (second-order moments of the beam distribution) is independent of the phase advance, and becomes a function of the envelope matrix only. These features significantly simplify the calculations of the beam envelopes. Recently, a new class of KV distributions were found by allowing arbitrary emittance combinations [11]. In this paper, we apply the formulation introduced in Ref. [11] for the description of the Gaussian distribution with unequal mode emittances. The Gaussian beam distributions with unequal mode emittances occur commonly in many accelerator systems, and thus they are of practical importance.

As an illustrative example, we apply this formulation to the analysis of continuouslyrotating quadrupole focusing channels. Rotating quadrupole focusing channels have been investigated by many authors [12-18] as they can potentially provide stronger focusing than conventional FODO lattices due to the dense population of the focusing elements [16]. For example, an electrostatic version of a continuously-rotating quadrupole focusing channel has been proposed for guiding very heavy charged particles [14]. Strong coupling in the rotating quadrupole system often induces beam rotation and reduces the beam pulsation [18]. We also note in this paper that beam propagation thorough a very long, continuously-rotating quadrupole focusing channel can be studied effectively in the laboratory frame of a linear Paul trap system with eight electrodes rather than four. 


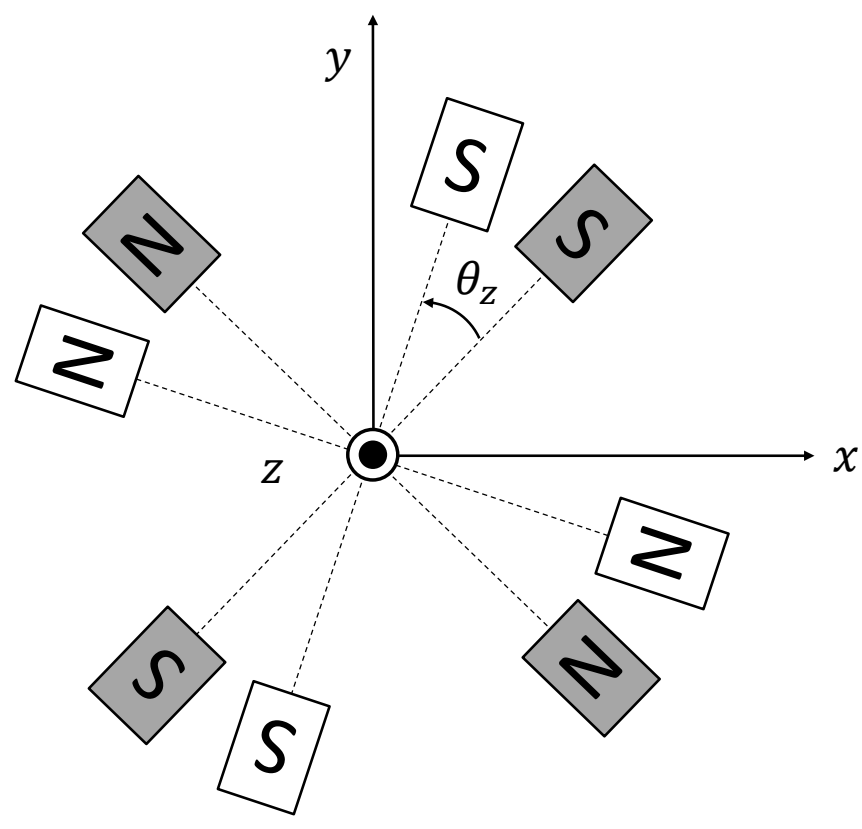

FIG. 1: Top view of the continuously rotating quadrupole focusing channel. Initial positions of the quadrupole magnets at $z=0$ are indicated by gray blocks. As the axial position along the $z$-axis increases, the initial quadrupole configuration rotates by an angle $\theta_{z}=2 \pi z / \lambda$ in the counterclockwise direction.

\section{CONTINUOUSLY-ROTATING QUADRUPOLE FOCUSING CONFIGURA- TIONS}

In this section, we introduce the field configuration for continuously-rotating quadrupole focusing channels, and derive the transverse orbit equations.

\section{A. Rotating quadrupole magnetic field}

Suppose near the beam axis that the quadrupole magnets generate the following field configuration at $z=0$ (see grey blocks in Fig. 1):

$$
\mathbf{B}_{q}(x, y, 0)=B_{q}^{\prime}(y \hat{x}+x \hat{y})
$$

where $B_{q}^{\prime}$ is the field gradient. When the quadrupole magnets are continuously rotating along the beam axis with an angle $\theta_{z}=2 \pi z / \lambda$ (see white blocks in Fig. 1) with respect to 
the initial configuration, the magnetic field is given by

$$
\mathbf{B}_{q}(x, y, z)=B_{q}^{\prime} \cos 2 \theta_{z}(y \hat{x}+x \hat{y})-B_{q}^{\prime} \sin 2 \theta_{z}(x \hat{x}-y \hat{y}) .
$$

Here, $\lambda$ is the periodicity of the pole configuration, which is twice the periodicity of the focusing field $L$, i.e., $\lambda=2 L$. In this channel configuration, the transverse orbit equations for a continuous beam propagating with average axial velocity $V_{b}=\beta_{b} c=$ const. are given by

$$
\begin{aligned}
& \frac{d^{2} x}{d z^{2}}+\kappa_{q m} \cos (2 \pi z / L) x+\kappa_{q m} \sin (2 \pi z / L) y=0 \\
& \frac{d^{2} y}{d z^{2}}+\kappa_{q m} \sin (2 \pi z / L) x-\kappa_{q m} \cos (2 \pi z / L) y=0
\end{aligned}
$$

where $\kappa_{q m}=e_{b} B_{q}^{\prime} / \gamma_{b} m_{b} \beta_{b} c$. Here, $\gamma_{b}=\left(1-\beta_{b}^{2}\right)^{-1 / 2}$ is the relativistic mass factor, $e_{b}\left(m_{b}\right)$ is the charge (rest mass) of a beam particle, and $c$ is the speed of light in vacuo. In practice, the continuously-rotating quadrupole focusing configuration can be realized by winding four wires (by alternating the current polarity from the adjacent wires) with helical symmetry $[12]$.

\section{B. Rotating quadrupole electric field}

By noting the well-known analogy between an intense ion beam propagating in a periodic focusing quadrupole magnetic field and a nonneutral single component charge bunch confined in a linear Paul trap [19], we can also realize the continuously-rotating quadrupole focusing configuration by means of time-varying electric fields. First, we consider an applied potential $\phi_{q}(r, \theta, t)$ that satisfies the boundary conditions at $r=r_{w}$ as follows (see black plates in Fig. 2):

$$
\phi_{q}\left(r_{w}, \theta, t\right)=\left\{\begin{array}{cc}
+V(t), & 0 \leq \theta<\frac{1}{8} \pi, \\
0, & \frac{1}{8} \pi \leq \theta<\frac{3}{8} \pi, \\
-V(t), & \frac{3}{8} \pi \leq \theta<\frac{5}{8} \pi, \\
0, & \frac{5}{8} \pi \leq \theta<\frac{7}{8} \pi, \\
+V(t), & \frac{7}{8} \pi \leq \theta \leq \pi .
\end{array}\right.
$$

We note that $\phi_{q}\left(r_{w}, \theta+\pi, t\right)=\phi_{q}\left(r_{w}, \theta, t\right)$. Neglecting end effects $(\partial / \partial z=0)$, it is readily shown that the solution to $\nabla_{\perp}^{2} \phi_{q}(x, y, t)=0$ with boundary conditions (5) is given by

$$
\phi_{q}=\frac{4 V(t)}{\pi} \sum_{l=1}^{\infty} \frac{\sin (l \pi / 2)}{l} \cos (l \pi / 4)\left(\frac{r}{r_{w}}\right)^{2 l} \cos (2 l \theta),
$$




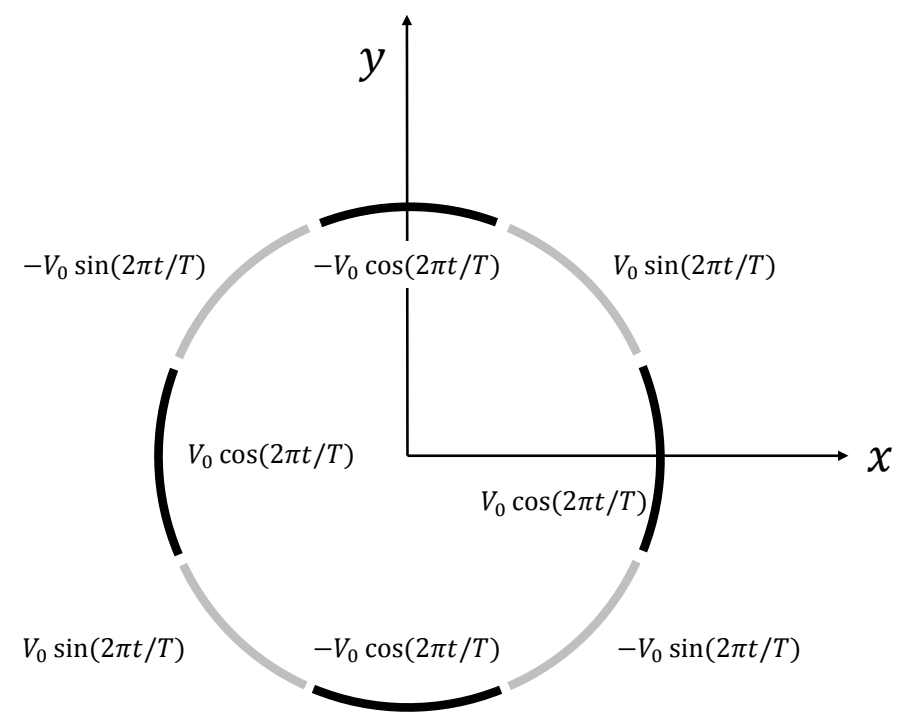

FIG. 2: Top view of the long $(\partial / \partial z=0)$ linear Paul trap configuration with eight electrodes. For the black (gray) plates, cosine (sine) voltage wavefroms are applied with alternating polarity on adjacent black (gray) segments.

for $0 \leq r \leq r_{w}$ and $0 \leq \theta \leq 2 \pi$. Near the cylinder axis $\left(r \ll r_{w}\right)$, Eq. (6) readily gives to lowest order

$$
\phi_{q}(x, y, t)=\frac{1}{2} \frac{m}{q} \kappa_{q e} \cos \left(\frac{2 \pi t}{T}\right)\left(x^{2}-y^{2}\right) .
$$

Here, we assume $V(t)=V_{0} \cos (2 \pi t / T)$ and the quadrupole focusing coefficient is defined by

$$
\kappa_{q e}=\frac{4 \sqrt{2} q V_{0}}{m \pi r_{w}^{2}},
$$

where $T$ is the period of the applied voltage and $q(m)$ is the charge (rest mass) of a trapped ion. Now, we rotate the configuration described in Eq. (5) by an angle $\pi / 4$ in the counterclockwise direction (see gray plates in Fig. 2), and apply $V(t)=V_{0} \sin (2 \pi t / T)$. This gives the skew component

$$
\phi_{s q}(x, y, t)=\frac{m}{q} \kappa_{q e} \sin \left(\frac{2 \pi t}{T}\right) x y .
$$


The single-particle equations of motions in the combination of both the quadrupole and skew-quadrupole potential configurations are

$$
\begin{aligned}
& \frac{d^{2} x}{d t^{2}}+\kappa_{q e} \cos (2 \pi t / T) x+\kappa_{q e} \sin (2 \pi t / T) y=0 \\
& \frac{d^{2} y}{d t^{2}}+\kappa_{q e} \sin (2 \pi t / T) x-\kappa_{q e} \cos (2 \pi t / T) y=0
\end{aligned}
$$

which are identical in functional form to the transverse orbit equations in Eqs. (3) and (4). We note that the Paul Trap Simulator Experiment (PTSX) device [20] at the Princeton Plasma Physics Laboratory (PPPL) could be easily converted into a dedicated experimental facility for studying coupled transverse dynamics in rotating quadrupole channels by subdividing its four electrodes into eight.

\section{GENERALIZED COURANT-SNYDER THEORY}

The general form of the Hamiltonian for the coupled transverse dynamics with quadrupole and skew-quadrupole focusing lattices is given by

$$
H_{c}=\frac{1}{2} X^{T} A_{c} X, A_{c}=\left(\begin{array}{cc}
\kappa(s) & R(s) \\
R(s)^{T} & I
\end{array}\right),
$$

where $X=\left(x, y, p_{x}, p_{y}\right)^{T}$. Here, the $2 \times 2$ matrix $\kappa(s)$ is time-dependent and symmetric, and $I$ is the $2 \times 2$ unit matrix. The variable $s$ plays the role of a time-like variable. For a beam propagating in a quadrupole magnetic focusing channel, we set $s=z$, while for the trapped single-component plasma confined in a linear Paul trap we set $s=t$. The prime denotes a derivative with respect to $s$ and the superscript " $T$ " denotes the transpose operation of a matrix. When there is a solenoidal field component, the $2 \times 2$ matrix $R$ is non-zero and defined by

$$
R(s)=\left(\begin{array}{cc}
0 & -\Omega(s) \\
+\Omega(s) & 0
\end{array}\right),
$$

where $\Omega(s)$ is the normalized Larmor frequency. Indeed, configurations with both axial magnetic fields and rotating quadrupole fields have been investigated for the modified betatron [21] and the spiral line induction accelerator (SLIA) [15]. In this paper, we consider only the case with $R=0$, for simplicity. Then, for the pure rotating quadrupole configuration, 
we have

$$
\kappa(s)=\kappa_{q 0}\left(\begin{array}{rr}
\cos (2 \pi s / L) & \sin (2 \pi s / L) \\
\sin (2 \pi s / L) & -\cos (2 \pi s / L)
\end{array}\right),
$$

where $L$ is the periodicity of the focusing field and $\kappa_{q 0}$ is the maximum quadrupole focusing strength, which is $\kappa_{q 0}=\kappa_{q m}$ for magnetic focusing, and $\kappa_{q 0}=\kappa_{q e}$ for electric focusing. The stability condition for the particle motion can be derived from an eigenvalue analysis [16], which gives

$$
0 \leq \kappa_{q 0} L^{2} \leq \pi^{2}
$$

Based on the generalized Courant-Snyder theory developed in Refs. [7, 8], we obtain the solution for the transverse dynamics governed by the Hamiltonian (12) in the form of a linear map:

$$
X(s)=M_{c} X_{0},
$$

where $X_{0}$ is the initial condition and $M_{c}$ is the transfer matrix given by

$$
M_{c}=Q^{-1} P^{-1} P_{0} Q_{0}
$$

Here, $P$ and $Q$ are $4 \times 4$ symplectic matrices defined by

$$
\begin{gathered}
P^{-1}=P^{T}=\left(\begin{array}{cc}
P_{1}^{T} & -P_{2}^{T} \\
P_{2}^{T} & P_{1}^{T}
\end{array}\right), P_{0}=\left(\begin{array}{ll}
I & 0 \\
0 & I
\end{array}\right), \\
P^{\prime}=P \phi^{\prime}, \phi^{\prime}=\left(\begin{array}{cc}
0 & -\left(w w^{T}\right)^{-1} \\
\left(w w^{T}\right)^{-1} & 0
\end{array}\right),
\end{gathered}
$$

and

$$
Q^{-1}=\left(\begin{array}{cc}
w^{T} & 0 \\
w^{\prime T} & w^{-1}
\end{array}\right), Q_{0}=\left(\begin{array}{cc}
\left(w^{-1}\right)^{T} & 0 \\
-w^{\prime} & w
\end{array}\right)_{0},
$$

where the subscript " 0 " denotes initial conditions at $s=0$. The symplectic matrix $P$ corresponds to a rotation in the $4 \mathrm{D}$ phase space $\left(P P^{T}=P^{T} P=I\right.$ and $\left.\operatorname{det}(P)=1\right)$, and thus $P \subset S p(4) \cap S O(4)=U(2)$. The $2 \times 2$ envelope matrix $w=\left(\begin{array}{ll}w_{1} & w_{2} \\ w_{3} & w_{4}\end{array}\right)$ is determined from the matrix envelope equation

$$
w^{\prime \prime}+w \kappa=\left(w^{-1}\right)^{T} w^{-1}\left(w^{-1}\right)^{T}
$$

This equation is the generalization of the familiar 1D envelope equation for an uncoupled focusing lattice. To make $Q$ symplectic, we require the following initial condition for $w$ :

$$
\left(w^{\prime} w^{T}-w w^{T}\right)_{0}=0
$$


For the uncoupled case, on the other hand, the initial condition (22) holds for any $w$. By solving the matrix envelope equation (21) numerically, one can describe the coupled transverse dynamics in a manner remarkably similar to the original Courant-Snyder theory.

\section{BEAM DISTRIBUTION WITH TWO EMITTANCES}

To describe a beam distribution $f(X)$ in $4 \mathrm{D}$ phase-space, we make the usual assumption that the phase-space density is a function of an invariant of motion $I_{C}$ which is expressed in the quadratic form $[6,22]$ :

$$
I_{C}=X^{T} \Xi X
$$

Here, $\Xi$ is a real, symmetric, and positive-definite matrix. Equation (23) represents a hyperellipsoid in 4D phase-space. To describe two independent normal modes and their emittances, it is desirable to diagonalize $\Xi$. Elementary linear algebra provides a simple way to transform a symmetric matrix into a diagonal form by solving an eigenvalue problem. However, the orthogonal matrices used in this diagonalization procedure are not generally symplectic. Therefore, they do not contain any physics principles of Hamiltonian dynamics. Note that the symplectic condition is a necessary and sufficient condition for a canonical transformation [23]. On the other hand, Williamson's theorem shows that any real, symmetric, and positive-definite matrix can be diagonalized in terms of a sympletic matrix $S$ [24] according to

$$
\Xi=S^{T}\left(\begin{array}{ll}
\Lambda & 0 \\
0 & \Lambda
\end{array}\right) S
$$

where

$$
\Lambda=\left(\begin{array}{cc}
\lambda_{1} & 0 \\
0 & \lambda_{2}
\end{array}\right),
$$

and the diagonal elements $\lambda_{j}$ are calculated from the characteristic equation, $\operatorname{det}\left(i J \Xi^{-1}-\right.$ $\left.\lambda_{j} I\right)=0$. The symplectic matrix $S$ allows a canonical transformation of coordinates into the normal forms, $\bar{X}=S X$. Using the usual convention, we define the mode emittances as $\epsilon_{1}=1 / \lambda_{1}$ and $\epsilon_{2}=1 / \lambda_{2}$.

There are several ways to find the symplectic transformation $S$. One is to construct $S$ using eigenvectors of the transfer matrix, which is well-established, for example in Ref. [6]. Another method, which is adopted here, is to use the generalized Courant-Snyder 
(CS) theory introduced in Sec. III. In the generalized CS theory, a remarkable canonical transformation $\bar{X}=S X=P Q X$ is constructed in such a way that $\bar{X}=\bar{X}_{0}=$ const. during the linear coupled motion. Therefore, for any constant and real $4 \times 4$ matrix $\xi$, which is symmetric and positive definite, an invariant in quadratic form can be constructed as $\bar{X}^{T} \xi \bar{X}=X^{T} S^{T} \xi S X=$ const.

To obtain the desired forms assumed in Eqs. (23) and (24), we consider the special case with

$$
\xi=\varepsilon^{-1}=\left(\begin{array}{cc}
\epsilon^{-1} & 0 \\
0 & \epsilon^{-1}
\end{array}\right), \epsilon=\left(\begin{array}{cc}
\epsilon_{1} & 0 \\
0 & \epsilon_{2}
\end{array}\right)=\Lambda^{-1} .
$$

This choice of $\xi$ allows $\bar{X}=\left(\bar{X}_{1}, \bar{X}_{2}, \bar{X}_{3}, \bar{X}_{4}\right)^{T}$ to become normalized coordinates describing a hyperellispoid in $4 \mathrm{D}$ phase-space with rotational symmetry in each normal plane:

$$
I_{C}=\frac{\bar{X}_{1}^{2}+\bar{X}_{3}^{2}}{\epsilon_{1}}+\frac{\bar{X}_{2}^{2}+\bar{X}_{4}^{2}}{\epsilon_{2}} .
$$

This is consistent with the basic assumption in beam physics, that due to phase mixing caused by unavoidable nonlinearities, the beam distribution in each normal plane becomes rotationally symmetric. For $\epsilon_{1}=\epsilon_{2}$, we have a hypersphere with full rotational symmetry.

The question may arise regarding the uniqueness of the canonical transformation $S$ giving the same emittances. It has been proven in Ref. [24] that the symplectic matrix $S$ diagonalizing $\Xi$ in Williamson's theorem [i.e., Eq. (24)] is unique up to a symplectic rotation. In other words, there is another symplectic matrix $\tilde{S}=C S$ which satisfies Eq. (24) with the same emittances, in which $C$ is an arbitrary constant $4 \times 4$ matrix in the unitary group $U(2)=S p(4) \cap S O(4)$. This property can be easily checked from Eq. (19) as well. If we multiply by the matrix $C$ on both sides of Eq. (19), note that $\tilde{P}=C P$ satisfies $\dot{\tilde{P}}=\tilde{P} \dot{\phi}$ with the new initial condition $\tilde{P}_{0}=C P_{0}=C$. Of course, $\tilde{P}$ is symplectic and $\tilde{P} \subset S O(4)$. Therefore, $\tilde{P} Q=C P Q=C S$ becomes another canonical transformation. This is consistent with the notion that the normalized coordinates in Eq. (27) admit the rotational symmetry in each normal plane.

It is worthwhile to note that the matrix envelope equation (21) admits an orthogonal symmetry. If $w$ is a solution of the Eq. (21), then $\tilde{w}=\tilde{c} w$ becomes a solution as well with $\tilde{c}$ being an arbitrary constant $2 \times 2$ orthogonal matrix, $\tilde{c}^{T} \tilde{c}=I$. With the new envelope 
matrix $\tilde{w}$, we obtain the corresponding $\tilde{P}$ and $\tilde{Q}$ as

$$
\tilde{P}=P\left(\begin{array}{cc}
\tilde{c}^{T} & 0 \\
0 & \tilde{c}^{T}
\end{array}\right), \tilde{Q}=\left(\begin{array}{cc}
\tilde{c} & 0 \\
0 & \tilde{c}
\end{array}\right) Q .
$$

Therefore, we obtain $\tilde{P} \tilde{Q}=P Q$, and thus the canonical transformation $S$ is independent of $\tilde{c}$.

Let's consider the case of a multivariate Gausssian distribution:

$$
f(X)=\frac{1}{(2 \pi)^{2} \sqrt{\operatorname{det}(\Sigma)}} \exp \left[-\frac{1}{2} X^{T} \Sigma^{-1} X\right]
$$

where $\Sigma=\Xi^{-1}=\left\langle X X^{T}\right\rangle$ is a covariance matrix (or the beam matrix in beam physics) and $\langle\cdots\rangle$ denotes the statistical average over the distribution. We assume $\langle X\rangle=0$ for simplicity. By comparing with Eq. (24), the expression for the beam matrix is given by

$$
\Sigma^{-1}=Q^{T} P^{T} \xi P Q
$$

and $\sqrt{\operatorname{det}(\Sigma)}=\sqrt{\operatorname{det}(\xi)}=\epsilon_{1} \epsilon_{2} \equiv \epsilon_{\perp}^{2}$. The distribution function $f(X)$ is normalized according to $\int \cdots \int f(X) d X_{1} \cdots d X_{4}=1$, which is a trivial result of the multivariate Gaussian integral. Often, it is important to project the beam distribution onto the spatial plane $z=\left(X_{1}, X_{2}\right)^{T}=(x, y)^{T}$. To perform the partial Gaussian integral, we express Eq. (30) in the following block form:

$$
Q^{T} P^{T} \xi P Q=\left(\begin{array}{cc}
U_{0} & V \\
V^{T} & W_{0}
\end{array}\right) .
$$

Based on the procedure in Ref. [25], it follows that

$$
\frac{1}{(2 \pi)^{2} \sqrt{\operatorname{det}(\Sigma)}} \iint \exp \left[-\frac{1}{2} X^{T} \Sigma^{-1} X\right] d X_{3} d X_{4}=\frac{\sqrt{\operatorname{det}(U)}}{2 \pi} \exp \left[-\frac{1}{2} z^{T} U z\right],
$$

where

$$
U=U_{0}-V W_{0}^{-1} V^{T}
$$

Since the expression for $Q$ is known explicitly from Eq. (20), it is sometimes more convenient to express $U$ in terms of the block matrix elements of $P^{T} \xi P$ :

$$
P^{T} \xi P=\left(\begin{array}{cc}
A & B \\
B^{T} & D
\end{array}\right) .
$$


Direct matrix manipulations that make use of Eqs. (31) and (34) show that

$$
U=w^{-1}\left[A-B D^{-1} B^{T}\right] w^{-T} \equiv w^{-1} R w^{-T}
$$

The $R$ matrix is known as the Schur complement of $D$ [11]. The $4 \mathrm{D}$ rms hyperellipsoid is projected onto a tilted ellipse given by

$$
z^{T} U z=z^{T} w^{-1} R w^{-T} z=1
$$

For the case of the equal emittances $\epsilon_{1}=\epsilon_{2}=\epsilon_{\perp}$, the phase advance terms in Eqs. (30) and (34) cancel out. Hence, the projection becomes

$$
z^{T} \beta^{-1} z=\epsilon_{\perp}
$$

and the beam matrix becomes

$$
\Sigma=\epsilon_{\perp}\left(\begin{array}{cc}
\beta & -\alpha \\
-\alpha^{T} & \gamma
\end{array}\right)
$$

where $\alpha, \beta$, and $\gamma$ are the generalized Twiss parameters defined as

$$
\begin{aligned}
& \alpha=-w^{T} w^{\prime}, \\
& \beta=w^{T} w \\
& \gamma=\left(w^{T} w\right)^{-1}+w^{\prime T} w^{\prime} .
\end{aligned}
$$

Note that the familiar relation between $\alpha, \beta$, and $\gamma$ holds in the matrix form:

$$
\beta \gamma=I+\alpha^{2}
$$

\section{NUMERICAL EXAMPLES}

In this section, we present numerical solutions of the matched beam envelopes in a continuously-rotating quadrupole focusing channel. Matched solutions are found by imposing periodic boundary conditions. First, we consider the case of equal mode emittances. As mentioned in the previous sections, in this case we don't need to solve the phase advance equation (19). Plotted in Fig. 3 is the evolution of the matched beam cross-section as a

function of the time-like variable $s$ for several different normalized focusing strengths $\kappa_{q 0} L^{2}$. 
The beam cross-section is the projection of the 4D rms hyperellipsoid, which is determined by the $\exp [-1 / 2]$ contour of the Gaussian beam distribution. It is clear that the matched beam cross-section rotates with the same periodicity as the focusing channel. This result is indeed the limiting case $(N \rightarrow \infty)$ of our previous work on the $N$-rolling lattice, which is a configuration with $N$ equally spaced quadrupole magnets, each of which rotates by an angle $\pi / N$ relative to its preceding magnet configuration [18]. As the values of $\kappa_{q 0} L^{2}$ increases, the beam cross-section becomes more elongated. Obviously, the rotation direction of the beam cross-section will reverse when the quadrupole configuration rotates in the opposite direction. Here, all the transverse dimensions are normalized by $\sqrt{\epsilon_{\perp} L}$.

The elongation, which is the ratio between the major axis $(a)$ and the minor axis $(b)$ of the beam cross-section, can be expressed analytically for the case with equal emittances as $[16]$

$$
a / b=\left[\frac{(2 \pi / L)^{2}+4 \kappa_{q 0}}{(2 \pi / L)^{2}-4 \kappa_{q 0}}\right]^{1 / 4}
$$

which is independent of $s$. This expression is derived from the eigenvalue analysis given in Ref. [16]. It is clear in Fig. 4 that the numerical values calculated from the generalized CS theory match almost perfectly with the analytical expression given in Eq. (43), which confirms the validity of the generalized CS theory.

Now we consider the cases of unequal mode emittances. Maintaining the 4D emittance $\epsilon_{\perp}^{2}$ constant, we vary the ratio between the two mode emittances $\epsilon_{1} / \epsilon_{2}$. This means that the volume of a hyperellispoid in 4D phase-space remains the same, whereas the shape changes according to the emittance ratio. As depicted in Figs. 5 and 6, the evolution of the matched beam cross-section become more complicated when the two mode emittances are different. The dynamics of the beam is not a simple rotation, but exhibits pulsation (periodic change in $a$ and $b$ ) as well. This results in a nonlinear increase of the tilt angle with time. The tilt angle is defined as the rotation angle of the major axis with respect to its initial position at $s=0$. For the case where $\epsilon_{1} / \epsilon_{2}=2$ (Fig. 5), the beam cross-section becomes more elongated in the $x$-direction. On the other hand, the beam cross-section becomes more elongated in the $y$-direction for $\epsilon_{1} / \epsilon_{2}=1 / 2$ (Fig. 6). The case of equal emittances corresponds to Figs. 3(a) and 3(b). Since the numerical matching algorithm now involves solutions of both the envelope and phase advance equations, which are highly nonlinear, errors can accumulate as we calculate the beam envelopes by numerical integration along $s$. For example, the tilt 
angle plotted in Fig. 5(d) does not return to its initial value but rather shows a slight offset at $s=L$. An improvement of the matching algorithm is being investigated for the case of unequal mode emittances.

\section{CONCLUSIONS}

In this paper, we have constructed the Gaussian beam distribution with two mode emittances in the context of the recently developed generalized CS theory of the coupled transverse dynamics. The formulation retains the elegant mathematical structure of the original CS theory with remarkably similar physical interpretations, and therefore can be viewed as complementary to other approaches such as the one based on eigenvectors of the transfer matrix [6]. As a numerical example, we apply this formulation to the case of a continuouslyrotating quadrupole focusing channel. Since some of the beam characteristics are known in terms of the analytical expressions for this channel, the formulation can be successfully benchmarked. The numerical calculations based on the present formulation not only agree very well with the results in Ref. [16], but also provide comprehensive information about the rotating beam. Furthermore, it has been demonstrated that the beam propagation thorough a very long, continuously-rotating quadrupole focusing channel can be studied equivalently in an experimental setup such as the linear Pual trap with 8-segmented electrodes. Finally, we emphasize that the formulation introduced in this paper can be readily applied to arbitrary (and more complex) linear, coupled focusing lattices other than continuously-rotating quadrupole channels.

\section{Acknowledgment}

This research was supported by the US Department of Energy.

[1] E. D. Courant and H. S. Snyder, Annals of Physics 3, 1 (1958).

[2] L. C. Teng, Fermi National Accelerator Laboratory Report FN-229 (1971).

[3] D. A. Edwards and L. C. Teng, IEEE Trans. Nucl. Sci. NS-20, 885 (1973).

[4] G. Ripken, Deutsches Elektronen-Synchrotron Internal Report R1-70/04 (1970). 
[5] I. Borchardt, E. Karantzoulis, H. Mais, and G. Ripken, Deutsches Elektronen-Synchrotron Internal Report 87-161 (1987).

[6] A. V. Lebedev and S. A. Bogacz, JINST 5, 10010 (2010).

[7] H. Qin and R. C. Davidson, Phys. Plasmas 16, 056701 (2009).

[8] H. Qin and R. C. Davidson, Phys. Rev. ST Accel. Beams 12, 064001 (2009).

[9] M. Chung, H. Qin, and R. C. Davidson, Phys. Plasmas 17, 084502 (2010).

[10] H. Qin, M. Chung, and R. C. Davidson, Phys. Rev. Lett. 103, 224802 (2009).

[11] H. Qin and R. C. Davidson, Phys. Rev. Lett. 110, 064803 (2013).

[12] K. J. L. Couteur, Plasma Physics 9, 457 (1966).

[13] H. O. Funsten, Nucl. Instrum. Meth. Phys. Res. A 44, 301 (1966).

[14] F. S. Chute, F. E. Vermeulen, and E. A. Youssef, Nucl. Instrum. Meth. Phys. Res. A 82, 86 (1970).

[15] C. M. Tang, P. Sprangle, J. Krall, P. Serafim, and F. Mako, NRL Memorandum Report 6589 (1990).

[16] A. Morita and Y. Iwashita, Phys. Rev. ST Accel. Beams 6, 014001 (2003).

[17] D. Toprek, Nucl. Instrum. Meth. Phys. Res. A 557, 397 (2006).

[18] H. Qin and R. C. Davidson, Phys. Plasmas 18, 056708 (2011).

[19] R. C. Davidson, H. Qin, and G. Shvets, Phys. Plasmas 7, 1020 (2000).

[20] E. P. Gilson, R. C. Davidson, P. C. Efthimion, and R. Majeski, Phys. Rev. Lett. 92, 155002 (2004).

[21] C. W. Roberson, A. Mondelli, and D. Chernin, Phys. Rev. Lett. 50, 507 (1983).

[22] Y. Alexahin, Muon Accelerator Program Document 4358-v1 (2013).

[23] H. Goldstein, Classical Mechanics (Addison-Wesley, Boston, 1980), 2nd ed.

[24] M. de Gosson and F. Luef, Physics Report 484, 131 (2009).

[25] E. T. Jaynes, Probability Theory:The Logic of Science (Washington University, St. Louis, 1995). 

(a) $\kappa_{q 0} L^{2}=4$ and $0 \leq s / L \leq 0.5$.
(b) $\kappa_{q 0} L^{2}=4$ and $0.5 \leq s / L \leq 1$.

(c) $\kappa_{q 0} L^{2}=6$ and $0 \leq s / L \leq 0.5$.

(d) $\kappa_{q 0} L^{2}=6$ and $0.5 \leq s / L \leq 1$.

$$
\text { (e) } \kappa_{q 0} L^{2}=8 \text { and } 0 \leq s / L \leq 0.5 . \quad \text { (f) } \kappa_{q 0} L^{2}=8 \text { and } 0.5 \leq s / L \leq 1 .
$$

FIG. 3: Evolution of the matched beam cross-section for $0 \leq s / L \leq 1$ with several different normalized focusing strengths $\kappa_{q 0} L^{2}$. The transverse dimensions are normalized by $\sqrt{\epsilon_{\perp} L}$. Colors of the boundary of the beam cross-section are blended according to $s / L$ from $\operatorname{Red}(s / L=0)$, to Green $(s / L=0.5)$ and Blue $(s / L=1)$. The time step between two consecutive ellipses is $\Delta s / L=1 / 16$. 
FIG. 4: Elongation of the matched beam cross-section plotted as a function of the normalized focusing strength $\kappa_{q 0} L^{2}$. The line is obtained from the analytical expression in Eq. (43) and the symbols represent numerical values calculated from the generalized Courant-Snyder theory. 

(a)For $0 \leq s / L \leq 0.5$.
(b)For $0.5 \leq s / L \leq 1$.

(c)Transverse dimensions. $\quad$ (d)Tilt angle.

FIG. 5: Evolution of the matched beam cross-section for $0 \leq s / L \leq 1$ with $\epsilon_{1}=\sqrt{2} \epsilon_{\perp}$ and $\epsilon_{2}=(1 / \sqrt{2}) \epsilon_{\perp}$. Here, we fix the normalized focusing strength $\kappa_{q 0} L^{2}$ to be 4 . The transverse dimensions are normalized by $\sqrt{\epsilon_{\perp} L}$. Colors of the boundary of the beam cross-section are blended according to $s / L$ from $\operatorname{Red}(s / L=0)$, to Green $(s / L=0.5)$ and Blue $(s / L=1)$. The time step between two consecutive ellipses is $\Delta s / L=1 / 16$. 

(a)For $0 \leq s / L \leq 0.5$.
(b)For $0.5 \leq s / L \leq 1$.

(c)Transverse dimensions. $\quad$ (d)Tilt angle.

FIG. 6: Evolution of the matched beam cross-section for $0 \leq s / L \leq 1$ with $\epsilon_{1}=(1 / \sqrt{2}) \epsilon_{\perp}$ and $\epsilon_{2}=\sqrt{2} \epsilon_{\perp}$. Here, we fix the normalized focusing strength $\kappa_{q 0} L^{2}$ to be 4 . The transverse dimensions are normalized by $\sqrt{\epsilon_{\perp} L}$. Colors of the boundary of the beam cross-section are blended according to $s / L$ from $\operatorname{Red}(s / L=0)$, to Green $(s / L=0.5)$ and Blue $(s / L=1)$. The time step between two consecutive ellipses is $\Delta s / L=1 / 16$. 